Tropical Journal of Pharmaceutical Research November 2021; 20 (11): 2249-2253

ISSN: $1596-5996$ (print); 1596-9827 (electronic) (C) Pharmacotherapy Group, Faculty of Pharmacy, University of Benin, Benin City, 300001 Nigeria

\title{
Effect of dexmedetomidine on miR-144-3p expression and epithelial mesenchymal transition in gastric cancer cells
}

\author{
Zong Chen ${ }^{1}$, Yong Ding ${ }^{1}$, Ying Zeng ${ }^{2}$, Zhifeng Chen ${ }^{1}$, Xueping Zhang ${ }^{3}$, Jianyan \\ Chen ${ }^{1,2 *}$ \\ ${ }^{1}$ Department of Anesthesiology, The First Affiliated Hospital of Guangdong Pharmaceutical University, Guangzhou; \\ ${ }^{2}$ Department of Anesthesiology, Shenzhen Hospital of integrated traditional Chinese and Western Medicine, Shenzhen; \\ ${ }^{3}$ Department of Anesthesiology, Shenzhen People's Hospital, The Second Clinical Medical College, Jinan University, Shenzhen \\ Anesthesiology Engineering Center, Shenzhen 518020, China
}

*For correspondence: Email: yan19o@163.com

Sent for review: 27 April 2021

Revised accepted: 6 October 2021

\begin{abstract}
Purpose: To investigate the effect of dexmedetomidine (DEX) on epithelial mesenchymal transition (EMT) in gastric cancer cells, and the role of microRNA-144-3p (miR-144-3p) in the process.

Methods: The effect of DEX on miRNA expression profile was analyzed using GEO database (https://www.ncbi.nlm.nih.gov/gds/). Human gastric cancer cells were cultured in vitro, and one group of cells was treated with saline for $48 \mathrm{~h}$ (control group). Cells treated with DEX at doses of $0.01,0.1$ and $1.0 \mu \mathrm{mol} / \mathrm{L}$ for $48 \mathrm{~h}$ were marked as low-, medium- and high-DEX concentration groups. The mRNA expression levels of miR-144-3p, ZEB1, E-cadherin and vimentin were determined using real-time quantitative polymerase chain reaction (RT-PCR), while the protein expressions of ZEB1, E-cadherin and vimentin were assayed with Western blotting. Cell proliferation was determined with CCK-8 assay, while metastasis was measured using Transwell assay.

Results: The GEO database demonstrated that the expression of miR-144-3p in rat cardiomyocytes was significantly decreased after DEX treatment $(p<0.05)$. The expression of miR-144-3p was decreased in all groups, when compared to the control group, but the expressions of ZEB1 and vimentin were increased, while that of E-cadherin was down-regulated $(p<0.05)$. Cell proliferation in the high$D E X$ concentration group was decreased $(p<0.05)$. The degrees of cell invasion and migration were increased in the medium- and high-DEX concentration groups $(p<0.05)$.

Conclusion: DEX promotes the metastasis of gastric cancer cells by regulation of epithelial mesenchymal transition (EMT) and the expression of miR-144-3p. This finding provides a new insight into the treatment of gastric cancer.
\end{abstract}

Keywords: Gastric cancer, Dexmedetomidine, E-cadherin, Vimentin, MiR-144-3p, Tumor metastasis, Epithelial mesenchymal transition

This is an Open Access article that uses a funding model which does not charge readers or their institutions for access and distributed under the terms of the Creative Commons Attribution License (http://creativecommons.org/licenses/by/4.0) and the Budapest Open Access Initiative (http://www.budapestopenaccessinitiative.org/read), which permit unrestricted use, distribution, and reproduction in any medium, provided the original work is properly credited.

Tropical Journal of Pharmaceutical Research is indexed by Science Citation Index (SciSearch), Scopus, International Pharmaceutical Abstract, Chemical Abstracts, Embase, Index Copernicus, EBSCO, African Index Medicus, JournalSeek, Journal Citation Reports/Science Edition, Directory of Open Access Journals (DOAJ), African Journal Online, Bioline International, Open-J-Gate and Pharmacy Abstracts

\section{INTRODUCTION}

Gastric cancer is second only to lung cancer as a leading cause of death in the world [1]. The onset of most gastric cancer cases is insidious, but they progress very rapidly. An understanding of the key factors that affect the malignant behavior of gastric cancer cells is very important guide in the clinical diagnosis and treatment of the disease. Dexmedetomidine (DEX), a frequently- 
used intravenous anesthetic, affects different biological processes in the body by regulating a variety of signal pathways $[2,3]$. The importance of DEX in malignant tumors has been progressively revealed. Many studies have indicated that DEX is involved in regulating the biological behavior of tumor cells [4,5]. However, the effect of DEX on gastric cancer cells, and its exact mechanism remain to be elucidated.

Micro-RNAs (miRNAs) are important posttranscriptional regulatory factors which participate in various biological processes, including the pathogenesis of malignant tumors $[6,7]$. In this study, bioinformatics methods and in vitro experiments were used to investigate the regulatory effect of DEX on gastric cancer cellrelated miRNA and malignant behavior, in order to provide a new insight into the use of DEX in malignant tumors.

\section{EXPERIMENTAL}

\section{Materials}

Dexmedetomidine was purchased from Absin Bioscience Inc (Shanghai, China), while RPMI1640 medium and Gibvco FBS were bought from ThermoFisher Scientific ThermoFisher Scientific (Shanghai, China). Trypsin was product of Invitrogen (Shanghai, China). Reverse transcription kit was obtained from Luoqi Biotechnology (Henan, China). Fluorescent dye SYBR Green I was purchased from Takara (Beijing, China). Protein lysate and protease inhibitor were products of Beyotime Biotechnology, (Shanghai, China). Antibodies for ZEB1, E-cadherin, Vimentin, $\beta$-actin were got from Proteintech Company (Proteintech, Wuhan, China), while Cell Counting Kit (CCK-8) was obtained from Topscience Co. Ltd (Shanghai, China).

\section{Cell culture}

HGC-27 cells (Shanghai Institute of Cell Biology, China, Shanghai) were cultured at $37{ }^{\circ} \mathrm{C}$ in $90 \%$ RPMI-1640 containing $10 \%$ FBS in a $5 \% \mathrm{CO}_{2}$ cell incubator at relative humidity $>95 \%$. Cells in logarithmic growth phase were divided into control group treated with normal saline, and three groups treated with DEX at doses of 0.01 , 0.1 and $1 \mu \mathrm{mol} / \mathrm{L}$. All treatments lasted for $48 \mathrm{~h}$.

\section{Quantitative real-time polymerase chain reaction (qRT-PCR)}

Total RNA was extracted from the cells in each group with TRIzol reagent. Then, the total RNA was reverse-transcribed to cDNA using reverse transcription kit (Takara, Japan) according the manufacturer's instructions. The primers for miR144-3p, ZEB1, E-cadherin, Vimentin and $\beta$-actin which were used for qPCR (Table 1) were synthesized by Sangon Biotech (Sangon, Shanghai, China). The qRT-PCR reaction system was constituted in line with the operating instructions in a 7500 real-time fluorescence quantitative PCR instrument (Applied Biosystems, American, CA). The reaction steps were as follows: $50{ }^{\circ} \mathrm{C}$ for $2 \mathrm{~min}, 95^{\circ} \mathrm{C}$ for $10 \mathrm{~min}$, $95{ }^{\circ} \mathrm{C}$ for $15 \mathrm{~min} \times 40$ cycles; $95^{\circ} \mathrm{C}$ for $15 \mathrm{sec}, 60$ ${ }^{\circ} \mathrm{C}$ for $1 \mathrm{~min}, 95{ }^{\circ} \mathrm{C}$ for $30 \mathrm{~min}$, and $60{ }^{\circ} \mathrm{C}$ for 15 sec. The mRNA expression levels of the genes were calculated using the $2^{-\Delta \Delta C t}$ method.

\section{Western blot assay}

Total protein was extracted with IP lysing solution containing protease inhibitor, and the protein concentration of the lysate was determined using BCA kit. Equal amounts of protein $(50-\mu \mathrm{g})$ were resolved on $10 \%$ polyacrylamide gel electrophoresis, followed by transfer to PVDF membrane. The membrane was blocked with 10 $\%$ skimmed milk at room temperature for $2 \mathrm{~h}$, prior to incubation with primary antibodies for ZEB1, E-cadherin and Vimentin (each at 1:1000 dilution) at $4{ }^{\circ} \mathrm{C}$ overnight. Thereafter, the membrane was incubated with secondary antibody at room temperature for $1 \mathrm{~h}$. The blots were subjected to enhanced chemiluminescence $(E C L)$ analysis for $1 \mathrm{~h}$. Image analysis was done with ChemiDoc XRS Chemiluminescence Imager (Bio-Rad, USA).

Table 1: Primer sequences for qRT-PCR

\begin{tabular}{lll}
\hline Gene & $\mathbf{5}^{\prime} \mathbf{- 3}$ & $\mathbf{3}^{\prime} \mathbf{- \mathbf { 5 } ^ { \prime }}$ \\
\hline miR-144-3p & GCGCGCTACAGTATAGATGATG & GCTGTCAACGATACGCTACG \\
ZEB1 & GCTTCTCACACTCTGGGTCTTA & CCTCATTCTCTGCCTCTTCTACC \\
E-cadherin & CCCGCCTTATGATTCTCTGCTCGTGTCC & TCCGTACATGTCAGCCAGCTTCTTGAA \\
Vimentin & TTTCTCCACGCCTCCAGTT & ATGTCTTCGGCCAGGTTGT \\
$\beta$-Actin & GGCAGGTCATCACCATCGG & CGTGTTGGCGTAGAGGTCTTT \\
\hline
\end{tabular}




\section{Determination of cell proliferation}

Cells in each group were inoculated in 96-well plate at $0,12,24,48$ and $72 \mathrm{~h}$, with 5 replicates at each time point. After adherence, $10 \mu \mathrm{L} \mathrm{CCK}$ 8 solution was added to each well. The wells were cultured in the dark for $2 \mathrm{~h}$, after which the optical density (OD) of each well was read at $450 \mathrm{~nm}$ in a microplate reader.

\section{Transwell assay}

Matrigel glue $(50 \mathrm{mg} / \mathrm{L})$ was diluted in serum-free RPMI-1640 medium at a volume ratio of $8: 1$. It was evenly spread in the center of Transwell chamber, and air-dried at $37{ }^{\circ} \mathrm{C}$ for $2 \mathrm{~h}$ as basement membrane reservoir. Cells in each group were digested, centrifuged and resuspended in RPMI-1640 containing $1 \%$ FBS in serum-free RPMI-1640 hydrated basement membrane. In each group, $3 \times 10^{4}$ cells were inoculated on the basement membrane as invasion model, while $1 \times 10^{4}$ cells were inoculated in Transwell chamber without Matrigel glue (migration model). After addition of $600 \mu \mathrm{L}$ of RPMI-1640 containing $10 \%$ FBS solution to the lower chamber, the cells were cultured in a constant temperature incubator for $24 \mathrm{~h}$. Thereafter, the cells were fixed with $4 \%$ paraformaldehyde for $15 \mathrm{~min}$, and stained with $0.1 \%$ crystal violet for $30 \mathrm{~min}$. Then, the cells were examined under a light microscope and counted using ImageJ software.

\section{Statistical analysis}

The SPSS 23.0 software was used for statistical analysis. Statistical differences in measurement data amongst the groups were analyzed using analysis of variance, while Student's $t$-test was used for pairwise comparison. Statistical significance was assumed at $p<0.05$.

\section{RESULTS}

Influence of DEX on miRNA expression profile

Results from GSE126106 chip analysis (https://www.ncbi.nlm.nih.gov/geo/query/acc.cgi? acc $=$ GSE126106) showed that the expressions of miR-871-5p and miR-881-3p were significantly up-regulated after DEX treatment, while the expressions of miR-451-5p and miR-144-3p were markedly reduced $(p<0.01)$. These results are presented in Figure 1.

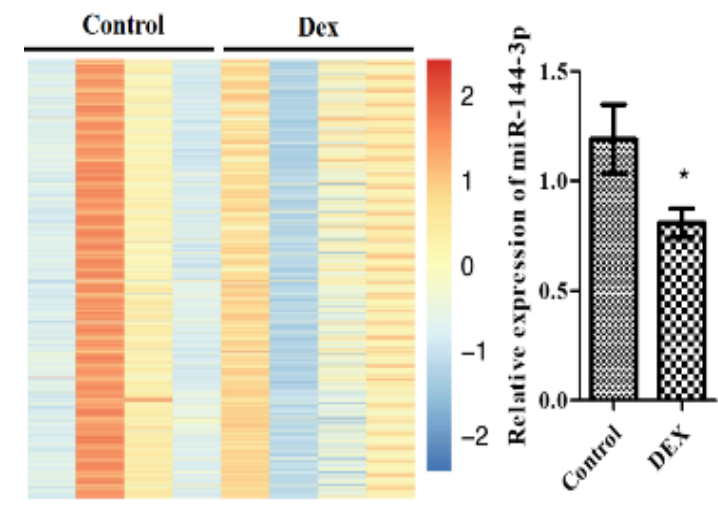

Figure 1: Effect of DEX on miRNA expression profiles in rat cardiomyocytes, as analyzed using GSE126106 chip analysis. ${ }^{\star} P<0.05$, compared to the DEX group

\section{Effect of DEX on the proliferation of HGC-27 cells}

The results of RT-qPCR and Western blot assays showed that, compared with the control group, the expressions of miR-144-3p in low, medium and high DEX concentration groups were decreased significantly, while the mRNA and protein expressions of ZEB1, vimentin were markedly up-regulated $(p<0.05$. Moreover, the expression of E-cadherin was significantly decreased $(p<0.05)$. These data are shown in Figure 2.

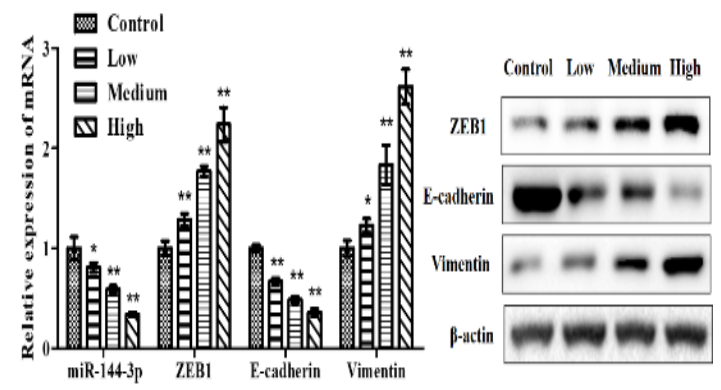

Figure 2: Expression levels of miR-133-3p and EMTrelated molecules in each group of cells. ${ }^{*} P<0.05$, compared to the control group; ${ }^{* *} p<0.001$, compared to the control group

\section{Effect of DEX on the proliferation of HGC-27 cells}

Compared with the control group, the proliferative potential of HGC-27 cells in high DEX concentration group was decreased significantly at $48 \mathrm{~h}$ and $72 \mathrm{~h}$, while there were no significant changes in cell proliferation at the low and middle DEX concentration groups $(p>$ 0.05 ; Figure 3 ). 


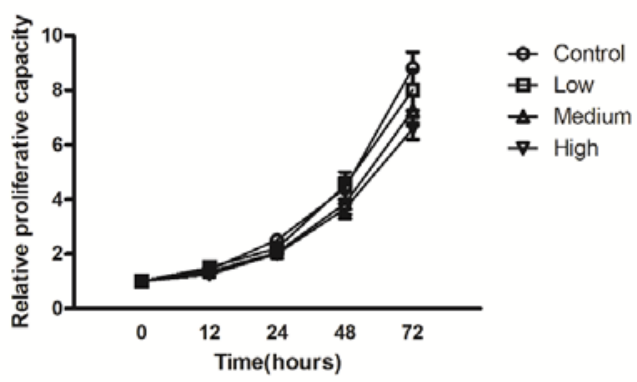

Figure 3: Cell proliferation potential in each group. ${ }^{* *} P$ $<0.001$, compared to the control group

\section{Effect of DEX on the invasion and migration of HGC-27 cells}

As shown in Figure 4, the invasion and migration potential of cells in the middle- and high-DEX concentration groups were significantly higher than those in the control group $(p<0.05)$.

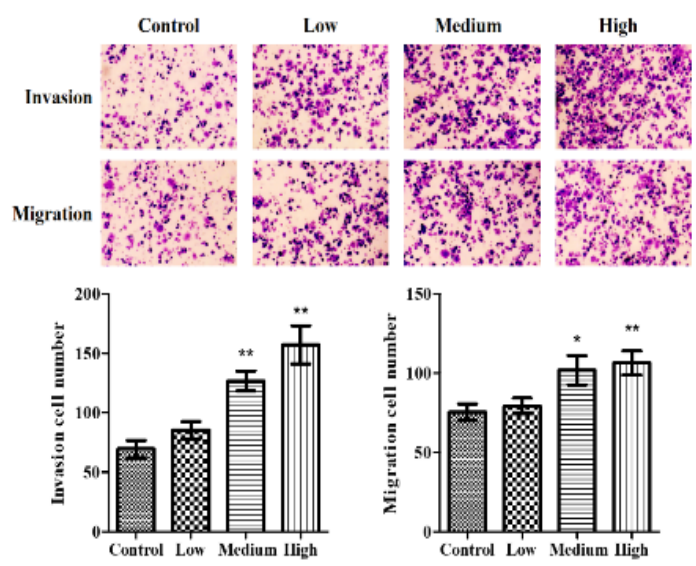

Figure 4: Cell invasion and migration potential in each group. ${ }^{\star} P<0.05$, compared to the control group; ${ }^{* *} p<$ 0.001 , compared to the control group

\section{DISCUSSION}

Dexmedetomidine (DEX) is a frequently-used intravenous anesthetic in clinics. In recent years, it has been discovered that DEX regulates the malignant behaviors of tumor cells. A study revealed that DEX significantly upregulated the phosphorylation levels of the transmembrane protease serine 2 (TMPRSS2) and STAT3 Tyr705 in breast cancer cells i.e., MCF-7 and MDA-MB-231, and promoted their migration [4]. Moreover, it has been reported that DEX enhanced the invasion and metastasis of A549 lung cancer cells and HCT116 colorectal cancer cells, through a mechanism related to upregulation of matrix metalloproteinases MMP-2 and MMP-9, and hypoxia-induced factor-1 $\alpha$ (HIF1a) [8]. Dexmedetomidine (DEX) up-regulated the expression of miR-155 in ovarian cancer cells, thereby inhibiting the tumor-promoting effect mediated by HIF-1 $\alpha$ [9]. It has also been demonstrated that DEX promoted the expression of miR-143-3p and inhibited the malignant behavior of esophageal cancer cells [10]. These results indicate that DEX exerts different effects on different malignant tumor cells. However, its effect on gastric cancer cells, and the related mechanisms are still unclear. In the present study, it was found that DEX significantly downregulated the expression level of miR-144-3p in rat myocardium in the GEO database chip (GSE126106). Thus, it can be reasonably speculated that miR-144-3p may serve as a potential target of DEX.

Low expression of miR-144-3p is often closely associated wth malignant tumors [11-13]. It has been reported that in gastric cancer, the expression level of miR-144-3p was significantly lower than those of adjacent tissues, and it was negatively correlated with lymph node metastasis and TNM stage, suggesting that miR-144-3p may be a protective factor for gastric cancer [14]. In vitro studies have shown that miR-144-3p promoted the radiosensitivity of gastric cancer cells by inhibiting the expression of zinc finger transcription factor ZEB1 [15]. Moreover, it has been reported that miR-144-3p regulated the target molecules PBX3 and CBX4, and inhibited epithelial-mesenchymal transformation, proliferation and metastasis of gastric cancer cells $[16,17]$.

In the present study, human gastric cancer cells HCG-27 were used as an in vitro model. It was found that the expression of miR-144-3p was significantly and dose-dependently reduced after DEX treatment. This finding was consistent with the sequencing data of the GSE126106 chip. At the same time, the expression of EMT-related molecules downstream of miR-144-3p were significantly changed, and the EMT process was enhanced. Moreover, the levels of invasion and migration were significantly increased. These results indicate that DEX inhibited the expression of miR-144-3p and promoted the EMT process of gastric cancer cells. Interestingly, cell proliferation was inhibited on treatment with the high concentration of DEX, due to the cytotoxicity produced the drug through other mechanisms.

\section{CONCLUSION}

DEX promotes the metastasis of gastric cancer cells by regulation of miR-144-3p and EMT. This finding provides a new insight into the treatment of gastric cancer. 


\section{DECLARATIONS}

\section{Acknowledgement}

This study was supported by the Shenzhen Municipal Commission of science and technology innovation (Grant no.JCYJ20170306144452663).

\section{Conflict of Interest}

No conflict of interest associated with this work.

\section{Contribution of Authors}

We declare that this work was done by the authors named in this article, and all liabilities pertaining to claims relating to the content of this article will be borne by the authors. Zong Chen and Yong Ding conceived and designed the study. Ying Zeng, Zhifeng Chen, Xueping Zhang and Jianyan Chen collected and analyzed the data, while Zong Chen wrote the manuscript which was approved by all authors. Zong Chen and Yong Ding contributed equally to this study.

\section{Open Access}

This is an Open Access article that uses a funding model which does not charge readers or their institutions for access and distributed under the terms of the Creative Commons Attribution License (http://creativecommons.org/licenses/by/ 4.0) and the Budapest Open Access Initiative (http://www.budapestopenaccessinitiative.org/rea d), which permit unrestricted use, distribution, and reproduction in any medium, provided the original work is properly credited.

\section{REFERENCES}

1. Bray F, Ferlay J, Soerjomataram I, Siegel RL, Torre LA, Jemal A. Global cancer statistics 2018: GLOBOCAN estimates of incidence and mortality worldwide for 36 cancers in 185 countries. CA Cancer J Clin 2018; 68(6): 394-424.

2. $Y u Z Y$, Geng J, Li ZQ, Sun YB, Wang SL, Masters J, Wang $D X$, Guo $X Y$, Li M, Ma D. Dexmedetomidine enhances ropivacaine-induced sciatic nerve injury in diabetic rats. Br J Anaesth 2019; 122(1): 141-149.

3. Zhao Y, He J, Yu N, Jia C, Wang S. Mechanisms of Dexmedetomidine in Neuropathic Pain. Front Neurosci 2020; 14: 330

4. Chi $M$, Shi $X$, Huo $X$, Wu $X$, Zhang $P$, Wang $G$. Dexmedetomidine promotes breast cancer cell migration through Rab11-mediated secretion of exosomal TMPRSS2. Ann Transl Med 2020; 8(8): 531.
5. Wang Z, Shen Z, Wang H, Zhang L, Dong R. Effect of dexmedetomidine on the cognitive function of patients undergoing gastric cancer surgery by regulating the PI3KJAKT signaling pathway. Oncol Lett 2020; 19(2): 1151-1156.

6. Wei L, Sun J, Zhang N, Zheng Y, Wang X, Lv L, Liu J, Xu $Y$, Shen $Y$, Yang M. Noncoding RNAs in gastric cancer: implications for drug resistance. Mol Cancer 2020; 19(1): 62

7. Zhou M, Wu Y, Li H, Zha X. MicroRNA-144: A novel biological marker and potential therapeutic target in human solid cancers. J Cancer 2020; 11(22): 67166726.

8. Chen HY, Li GH, Tan GC, Liang H, Lai XH, Huang $Q$, Zhong JY. Dexmedetomidine enhances hypoxiainduced cancer cell progression. Exp Ther Med 2019; 18(6): 4820-4828.

9. Zheng L, Jia R, Zhao J. Dexmedetomidine Regulates Proliferation, Apoptosis, Migration, and Invasion in Ovarian Cancer Cells via MiR-155-HIF-1a Axis. Med Sci Monit 2019; 25: 10164-10172.

10. Zhang $P, H e$, Bai Y, Liu W, Huang $L$. Dexmedetomidine suppresses the progression of esophageal cancer via miR-143-3p/epidermal growth factor receptor pathway substrate 8 axis. Anticancer Drugs 2020; 31(7): 693-701.

11. Wu J, Zhao Y, Li F, et al. MiR-144-3p: a novel tumor suppressor targeting MAPK6 in cervical cancer. J Physiol Biochem 2019; 75(2): 143-152.

12. Sun N, Zhang L, Zhang C, Yuan Y. miR-144-3p inhibits cell proliferation of colorectal cancer cells by targeting $B C L 6$ via inhibition of Wnt/beta-catenin signaling. Cell Mol Biol Lett 2020; 25: 19.

13. Chen YJ, Guo YN, Shi K, Huang HM, Huang SP, XuWQ, Li ZY, Wei KL, Gan TQ, Chen G. Down-regulation of microRNA-144-3p and its clinical value in non-small cell lung cancer: a comprehensive analysis based on microarray, miRNA-sequencing, and quantitative realtime PCR data. Respir Res 2019; 20(1): 48.

14. Sahami-Fard MH, Yazd EF, Jamalpoor Z, Nezhadi A. Expression Levels of miR-127-3p and miR-144-3p in Gastric Cancer and their Relationships with Clinicopathological Parameters. Clin Lab 2020; 66(6): 417.

15. Gao ZY, Liu H, Zhang Z. miR-144-3p increases radiosensibility of gastric cancer cells by targeting inhibition of ZEB1. Clin Transl Oncol 2020; 7: 618.

16. Li B, Zhang S, Shen $H, L i$ C. MicroRNA-144-3p suppresses gastric cancer progression by inhibiting epithelial-to-mesenchymal transition through targeting PBX3. Biochem Biophys Res Commun 2017; 484(2): 241-247.

17. Yang Z, OuYang X, Zheng L, Dai L, Luo W. Long intergenic noncoding RNA00265 promotes proliferation of gastric cancer via the microRNA-144-3p/Chromobox 4 axis. Bioengineered 2021; 12(1): 1012-1025. 